\title{
High-temperature stress susceptibility of representative japonica rice cultivars derived from Norin-22: Inadequate ATP supply during seed development may lead to severe damage
}

\author{
Kao-Chih She1,2, Mitsuhiro Yaeshima1, Takemasa Koumoto', Mariko Ohnuma1, \\ Tomoko Hiromasa ${ }^{1}$, Mio Hirai ${ }^{1}$, Takuya Matsunaga', Ryouka Tashiro', \\ Tadamasa Sasaki ${ }^{1}$, Hiroaki Kusano ${ }^{1}$, Hiroaki Shimada ${ }^{1,2, *}$ \\ ${ }^{1}$ Department of Biological Science and Technology, Tokyo University of Science, Noda, Chiba 278-8510, Japan; ${ }^{2}$ Reaserch \\ Institute for Science \& Technology, Tokyo University of Science, Noda, Chiba 278-8510, Japan \\ *E-mail: shimadah@rs.noda.tus.ac.jp Tel: +81-4-7124-1501 Fax: +81-4-7122-1499
}

Received August 24, 2012; accepted October 15, 2012 (Edited by M. Sekine)

\begin{abstract}
High temperature stress during seed development can reduce the rice grain yield and lead to poor milling quality because of inadequate grain filling. We tested the high temperature stress tolerance of eight rice cultivars and fluctuations in the ATP content during seed development. The phenotype of Norin-22 was very sensitive to high temperature conditions, which decreased the grain yield and produced defective characteristics. A significant reduction in the ATP content was also detected in the developing seeds. Koshihikari and Nipponbare, which are progenies derived from Norin-22, are known to be sensitive to high temperature stress. It is suggested that the high temperature stress sensitivity of Norin-22 was inherited by these cultivars. Shinriki was tolerant of high temperature stress, and it produced a large proportion of normal-shaped grains under high temperature stress conditions, with only a moderate decrease in its ATP content. These results suggest that there was a relationship between ATP deficiency and defective endosperm characteristics. Therefore, it is expected that identifying a rice cultivar that produces an adequate amount of ATP during seed development will be useful for breeding a cultivar that tolerates high temperature stress.
\end{abstract}

Key words: ATP content, developing rice seeds, high temperature ripening, inadequate grain filling, white core endosperm.

Grain production is sensitive to abiotic stresses such as high or low temperatures, submergence, and desiccation. Future global warming may lead to high temperature stress during the grain-filling stage of rice seed development, which will reduce the crop yield and quality (Shirato et al. 2007). In rice fields, a high temperature environment during seed development leads to many changes in the characteristics of rice grains, such as a chalky texture, milky appearance, and reduced grain weight (Yamakawa et al. 2007). These changes are caused by the formation of large airspaces in the endosperm because of inadequate growth of starch granules (Tashiro and Wardlaw 1991; Zakaria et al. 2002).

If rice seeds develop in high temperature conditions, the expression of multiple genes, such as those responsible for starch biosynthesis in the endosperm, is repressed; this results in a decreased amylose content and an aberrant amylopectin structure (Yamakawa et al.
2007). Thus, starch deposition may be impaired because of the downregulation of sucrose import/degradation and starch biosynthesis, and/or the upregulation of starch degradation, and inefficient ATP production because of inhibition of the cytochrome respiratory chain (Yamakawa et al. 2010).

The phenotype of the rice flo2 mutant grain is milky and floury, which is very similar to the chalky endosperm produced under high temperature conditions. The expression of genes involved in storage starch and storage protein biosynthesis is greatly reduced in the flo 2 endosperm (She et al. 2010b). Thus, the appearance of this phenotype is strongly correlated with a lower level of ATP synthesis in seeds ripening at high temperature and in the developing flo2 mutant seeds (She et al. 2010a). These observations suggest that adequate ATP synthesis is essential for efficient grain filling, which supports a hypothesis of the reduced production of storage

Abbreviations: ATP, adenosine triphosphate; DAF, days after flowering. 
substances in rice grains at high temperature.

Previously, we determined the relationship between ATP concentration in developing seeds and the efficiency of grain filling in representative rice cultivars (She et al. 2010a). We found that the cultivars Nipponbare and Taichung-65 were severely affected by high temperature stress, which inhibited their grain filling (She et al. 2010a). We also found that the rice cultivar Kimmaze was tolerant of high temperature stress (She et al. 2010a). These observations showed that there are differences in the high temperature stress tolerance of rice cultivars. Thus, high temperature stress-tolerant cultivars may be present in the pool of rice cultivars.

In this study, we tested the potential high temperature stress tolerance of representative rice cultivars. We also analyzed the progress of grain filling and fluctuations in the ATP content of developing seeds under normal or high temperature conditions using various rice cultivars. Thus, we report the tolerance of rice cultivars to high temperature stress and the relationship between the ATP supply during seed development and efficient grain filling.

\section{Materials and methods}

\section{Rice cultivars, and growth condition}

The Oryza sativa L. japonica rice cultivars used in this study, i.e., Aichi-asahi, Fukumasari, Ginbouzu, Norin-1, Norin-8, Norin-22, and Shinriki, were provided by the Rice Genetic Resource Center, Tsukuba, Japan. A japonica rice cultivar Nikomaru was provided from Kyushu Okinawa Agricultural Research Center, National Agriculture and Food Research Organization, Kumamoto, Japan. Plants were grown in pots in the open air during the summer. After flowering, the rice plants were cultivated in the growth chamber with a $12 \mathrm{~h} / 12 \mathrm{~h}$ day/ night cycle at $28^{\circ} \mathrm{C} / 25^{\circ} \mathrm{C}$ condition. In the high temperature treatment, the plants were temporarily moved to a growth chamber between 5 days after flowering (DAF) and 15 DAF, where they were grown with a $12 \mathrm{~h} / 12 \mathrm{~h}$ day/night cycle at $33^{\circ} \mathrm{C} / 28^{\circ} \mathrm{C}$ with constant $70 \%$ humidity, according to the method of Yamakawa et al. (2007). In the control experiment with normal temperature conditions, the plants were grown in a growth chamber throughout the seed development with a $12 \mathrm{~h} / 12 \mathrm{~h}$ day/night cycle at $28^{\circ} \mathrm{C} / 25^{\circ} \mathrm{C}$ with constant $70 \%$ humidity. The immature seeds were harvested at $5,7,10,15$, 20 , and 25 DAF. The seeds were frozen immediately using liquid nitrogen and stored in a $-80^{\circ} \mathrm{C}$ deep freezer until further analysis. The mature seeds were harvested at $40 \mathrm{DAF}$. Morphological changes were determined by measuring the seed size and weight. White core and other endosperm phenotypes were determined by illumination using a backlight.

\section{ATP measurement}

The ATP content of immature seeds was measured using the luciferase assay system where luciferin is converted to oxyluciferin reaction (Kimmich et al. 1975), using a Lucifer 250-plus kit (Kikkoman Co., Japan). The luminescent intensity of oxyluciferin was detected using an ARVOTM Light luminometer (PerkinElmer, USA). The weight of each immature seed was measured. Each seed was then powdered in liquid nitrogen using an AutoMill TK-AM5 (Tokken Inc. Japan) and suspended in the ATP extraction buffer from the Lucifer 250-plus kit.

Table 1. Proportion of defective grain produced in normal and high temperature conditions by eight rice cultivars.

\begin{tabular}{|c|c|c|c|c|c|c|c|c|}
\hline \multirow{2}{*}{ Grain feature } & \multicolumn{2}{|c|}{ Shinriki } & \multicolumn{2}{|c|}{ Aichi-asahi } & \multicolumn{2}{|c|}{ Norin-1 } & \multicolumn{2}{|c|}{ Nikomaru } \\
\hline & Normal & High & Normal & High & Normal & High & Normal & High \\
\hline Perfect grain & 96.0 & 76.0 & 96.0 & 45.5 & 96.2 & 40.0 & 91.3 & 38.1 \\
\hline Basal white & 0 & 12.0 & 4.0 & 0 & 0 & 28.0 & 0 & 28.6 \\
\hline White back & 0 & 12.0 & 0 & 40.9 & 0 & 32.0 & 4.3 & 14.3 \\
\hline White belly & 4.0 & 4.0 & 0 & 31.8 & 3.8 & 16.0 & 0 & 4.8 \\
\hline White core & 0 & 8.0 & 0 & 13.6 & 0 & 12.0 & 4.3 & 9.5 \\
\hline Milky white & 0 & 0 & 0 & 0 & 0 & 0 & 0 & 14.3 \\
\hline \multirow{2}{*}{ Grain feature } & \multicolumn{2}{|c|}{ Norin-8 } & \multicolumn{2}{|c|}{ Fukumasari } & \multicolumn{2}{|c|}{ Ginbozu } & \multicolumn{2}{|c|}{ Norin-22 } \\
\hline & Normal & High & Normal & High & Normal & High & Normal & High \\
\hline Perfect grain & 95.8 & 30.4 & 82.6 & 22.7 & 100 & 21.7 & 92.3 & 4.2 \\
\hline Basal white & 0 & 17.4 & 13.0 & 36.4 & 0 & 0 & 7.7 & 25.0 \\
\hline White back & 4.2 & 34.8 & 0 & 50.0 & 0 & 30.4 & 0 & 50.0 \\
\hline White belly & 0 & 4.3 & 0 & 9.1 & 0 & 4.3 & 0 & 8.3 \\
\hline White core & 0 & 26.1 & 4.3 & 13.6 & 0 & 26.1 & 0 & 41.7 \\
\hline Milky white & 0 & 0 & 0 & 4.5 & 0 & 30.4 & 0 & 25.0 \\
\hline
\end{tabular}

The proportion of grains with defective characteristics is indicated. For this analysis we used randomly selected 25 grains that were harvested from three individual pots of each line. Values are expressed as a percentage of the total grain. The sum of the values was not $100 \%$ because several grains exhibited two or more characteristics. "Normal" and "High" indicate the results for grains grown in normal and high temperature conditions, respectively. 
A
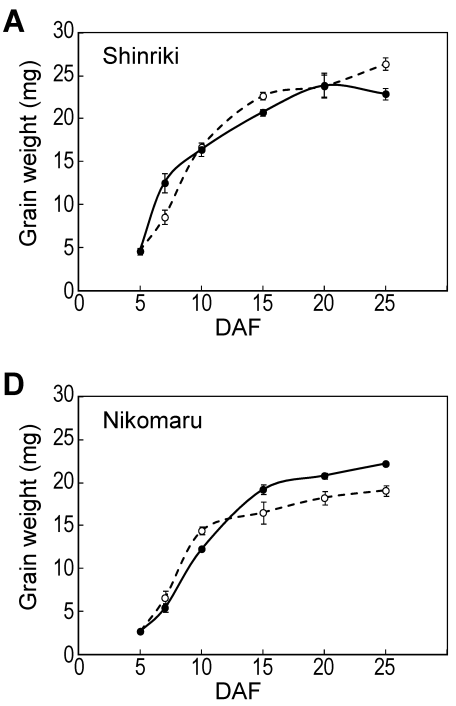

$G$

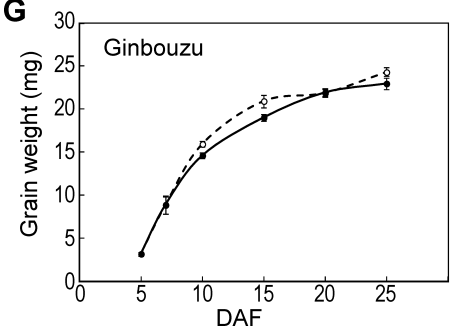

B

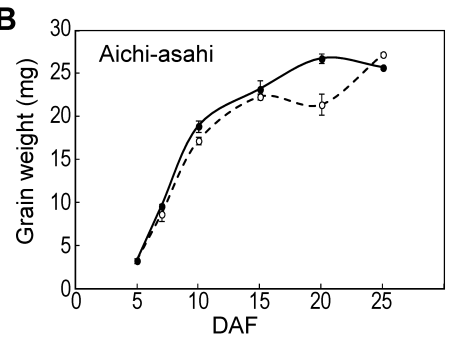

E

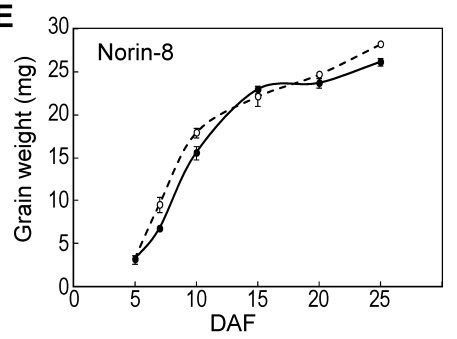

H

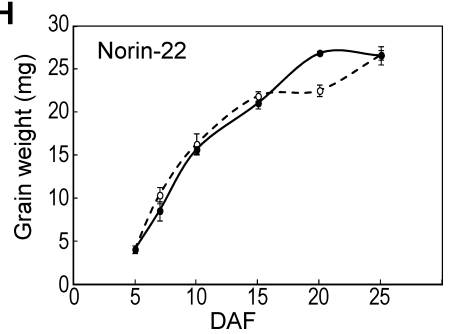

C

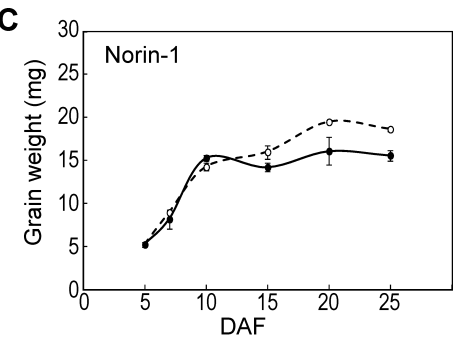

$\mathbf{F}$

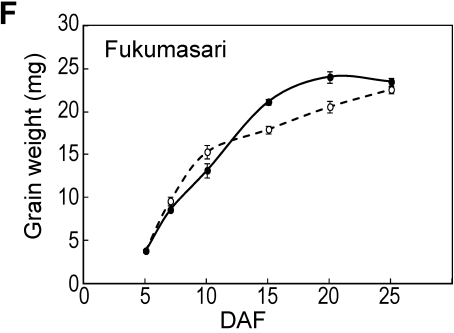

- - - Normal Temperature

- - High Temperature

Figure 1. Time course of seed enlargement during seed development in normal and high temperature conditions. Fresh weights of the seeds were measured using six immature seeds from Shinriki (A), Aichi-asahi (B), Norin-1 (C), Nikomaru (D), Norin-8 (E), Fukumasari (F), Ginbouzu (G), and Norin-22 (H) at 5, 7, 10, 15, 20, and 25 days after flowering (DAF). The open and filled circles represent seeds grown in normal and high temperature conditions, respectively. The error bars represent the standard errors.

\section{Results}

\section{Comparison of the characteristics of rice grains ripened at high temperature}

We evaluated the high temperature stress tolerance of eight candidate rice cultivars, i.e., Aichi-asahi, Fukumasari, Ginbouzu, Nikomaru, Norin-1, Norin-8, Norin-22, and Shinriki. All eight cultivars produced normal-shaped, good quality grains when grown in normal conditions (Table 1). By contrast, when grown under high temperature conditions, the rice grains of many of these cultivars exhibited major defective characteristics such as having basal white and white back grains, a chalky area on the dorsal and proximal sides, or white core endosperm (Table 1).

Norin-22 was highly susceptible to high temperature stress and the proportion of normal grains was quite low with this cultivar (4.2\%). Most grains exhibited insufficient grain filling with severe defective phenotypes. The most severely affected phenotype, milky white grains, was present in $25.0 \%$ of grains (Table 1). In addition, basal white, white back, white belly, white core, and milky white features were detected frequently in the same samples. These results indicate that Norin-22 is very sensitive to high temperature stress during seed development.

By contrast, Shinriki had a relatively good grainfilling performance when the grain developed in high temperature condition, i.e., $76.0 \%$ of the grain was perfect. No milky white grains were detected, although the phenotypes of some grains were clearly affected by insufficient grain filling (Table 1). This shows that the cultivar Shinriki has a greater tolerance for high temperature stress.

In the other cultivars, the effect of high temperature stress depended on the cultivar. The proportion of perfect grains produced by cultivars Aichi-asahi, Norin-1, Nikomaru, Norin-8, Fukumasari, and Ginbouzu were $45.5 \%, 40.0 \%, 38.1 \%, 30.4 \%, 22.7 \%$, and $21.7 \%$, respectively (Table 1 ). These results demonstrated the great diversity of high temperature stress tolerance among rice cultivars.

\section{Seed development under normal or high temperature conditions}

We analyzed the grain-filling process in the developing seeds of the eight cultivars and compared them at high and normal temperatures. Although it was observed that the grain-filling process was slightly inhibited by high temperature stress in Norin-1 seeds and enhanced in 
Nikomaru and Fukumasari seeds, other cultivars showed similar grain-filling processes in both conditions (Figure 1). The seed size was smaller when Shinriki, Ginbouzu, and Norin-22 seeds were grown under high temperature conditions, whereas there was no significant reduction in the grain size of other cultivars (Table 2).

\section{Fluctuations in the ATP content of developing seeds during grain filling with high temperature stress}

The ATP content of the developing seeds was measured at $5,7,10,15,20$, and $25 \mathrm{DAF}$ in the grains of the eight cultivars, which were grown in normal and high temperature conditions. Overall, a higher level of ATP content was maintained in the developing seeds under the normal conditions during the middle stage of seed development (7-20 DAF) and later (Figure 2). In these conditions, the ATP content had two peaks at 10 DAF and 20 DAF when active starch storage occurred (Figure 2). The ATP content fluctuated throughout the development stages and there were differences among the cultivars. In particular, the ATP content of the Nikomaru grains was $>0.16 \mu \mathrm{molg}^{-1}$ (Figure 2D), which suggested that Nikomaru had the potential to produce a high ATP supply in the developing seeds.

By contrast, the ATP content was lower throughout the development stages in seeds grown in high temperature conditions (Figure 2). However, the grains of Shinriki maintained a relatively higher ATP content compared with the others throughout the seed development stages (Figures 2A, 2C). Two peaks of the ATP content were also observed in the grain-filling process of Shinriki seeds (Figure 2). The grains of other cultivars had a lower ATP content during the middle stage and later stages of seed development. In particular, the ATP content of the Norin-22, Norin-8 and Fukumasari grains was significantly lower when the seeds were grown in high temperature stress conditions. The ATP content of these grains declined rapidly after 15 DAF (Figures $2 \mathrm{G}, 2 \mathrm{H}$ ), suggesting that high temperature stress severely impaired the ATP supply in these developing seeds.

\section{Discussion}

We evaluated the grain-filling profiles of eight rice cultivars grown in the high temperature conditions. The experiments detected clear difference in the high temperature stress tolerance of individual cultivars.

Norin-22 was most sensitive to high temperature stress among the cultivars evaluated. A large proportion of the Norin-22 grains grown in high temperature conditions comprised small grains with defective characteristics caused by insufficient grain filling (Table 1). A similar phenomenon was reported in Koshihikari and its derivatives (Terashima et al. 2001), which are commonly cultivated in Japan because of their good taste and high quality texture. Koshihikari is a cross between Norin-1 and Norin-22 (Figure 3). Thus, Norin-22 is one of the

Table 2. Grain sizes of eight cultivars grown in normal and high temperature conditions.

\begin{tabular}{|c|c|c|c|c|c|c|}
\hline & \multicolumn{3}{|c|}{ Length (mm) } & \multicolumn{3}{|c|}{ Width (mm) } \\
\hline & Normal & High & $R$ & Normal & High & $R$ \\
\hline Shinriki & $5.27 \pm 0.03$ & $5.19 \pm 0.04$ & $-1.55 \%$ & $3.11 \pm 0.02$ & $2.98 \pm 0.04$ & $-3.99 \%$ \\
\hline Aichi-asahi & $5.29 \pm 0.05$ & $5.41 \pm 0.04$ & $2.17 \%$ & $3.12 \pm 0.03$ & $3.19 \pm 0.02$ & $2.07 \%$ \\
\hline Norin-1 & $5.03 \pm 0.04$ & $4.92 \pm 0.03$ & $-2.11 \%$ & $2.80 \pm 0.02$ & $2.82 \pm 0.02$ & $0.50 \%$ \\
\hline Nikomaru & $5.02 \pm 0.04$ & $4.96 \pm 0.04$ & $-1.20 \%$ & $2.78 \pm 0.04$ & $2.75 \pm 0.06$ & $-0.85 \%$ \\
\hline Norin-8 & $5.46 \pm 0.05$ & $5.47 \pm 0.04$ & $0.13 \%$ & $3.01 \pm 0.02$ & $3.09 \pm 0.02$ & $2.83 \%$ \\
\hline Fukumassari & $5.41 \pm 0.03$ & $5.15 \pm 0.04$ & $-4.78 \%$ & $2.86 \pm 0.02$ & $2.80 \pm 0.04$ & $-2.20 \%$ \\
\hline Ginbouzu & $4.73 \pm 0.04$ & $4.87 \pm 0.05$ & $2.85 \%$ & $3.04 \pm 0.02$ & $2.93 \pm 0.02$ & $-3.48 \%$ \\
\hline \multirow[t]{3}{*}{ Norin-22 } & $5.55 \pm 0.12$ & $5.49 \pm 0.06$ & $-0.94 \%$ & $2.94 \pm 0.03$ & $2.86 \pm 0.04$ & $-2.71 \%$ \\
\hline & \multicolumn{3}{|c|}{ Depth (mm) } & \multicolumn{3}{|c|}{ Weight (mg) } \\
\hline & Normal & High & $R$ & Normal & High & $R$ \\
\hline Shinriki & $2.24 \pm 0.01$ & $2.18 \pm 0.02$ & $-2.33 \%$ & 25.25 & 23.72 & $-6.08 \%$ \\
\hline Aichi-asahi & $2.18 \pm 0.02$ & $2.33 \pm 0.02$ & $7.06 \%$ & 25.35 & 27.47 & $8.35 \%$ \\
\hline Norin-1 & $1.95 \pm 0.01$ & $2.01 \pm 0.02$ & $2.98 \%$ & 18.58 & 18.82 & $1.29 \%$ \\
\hline Nikomaru & $2.07 \pm 0.03$ & $2.10 \pm 0.02$ & $1.36 \%$ & 19.78 & 20.11 & $1.67 \%$ \\
\hline Norin-8 & $2.18 \pm 0.02$ & $2.28 \pm 0.01$ & $4.35 \%$ & 25.58 & 25.84 & $1.00 \%$ \\
\hline Fukumassari & $2.01 \pm 0.02$ & $2.17 \pm 0.02$ & $7.94 \%$ & 21.09 & 22.23 & $5.39 \%$ \\
\hline Ginbouzu & $2.29 \pm 0.02$ & $2.25 \pm 0.02$ & $-1.72 \%$ & 22.75 & 22.44 & $-1.40 \%$ \\
\hline Norin-22 & $2.20 \pm 0.01$ & $2.24 \pm 0.03$ & $2.08 \%$ & 25.51 & 23.99 & $-5.96 \%$ \\
\hline
\end{tabular}

The length, width, depth, and weights of the grains are shown. The grain weight was the averaged value for 25 grains. All values other than the grain weight include the standard errors, which were determined using 25 grains. "Normal" and "High" are the results for grains grown in the normal and high temperature conditions, respectively. " $R$ " indicates the ratio (\%) of the values in the high temperature conditions to those of the normal temperature conditions. 
A

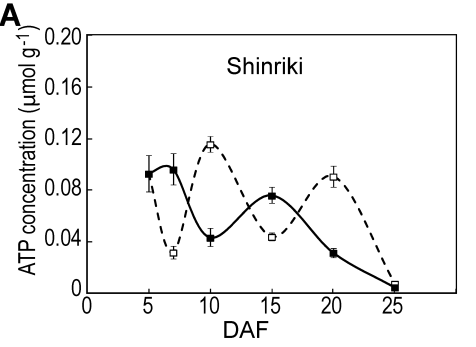

$D_{-}$

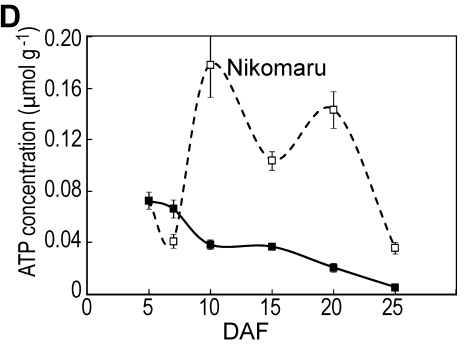

G

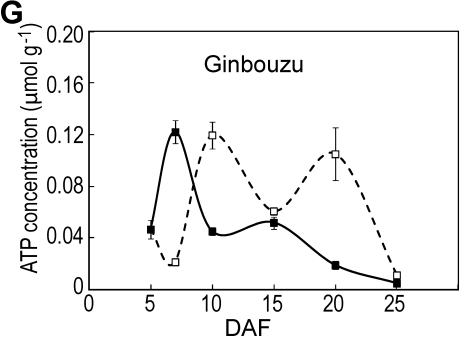

B

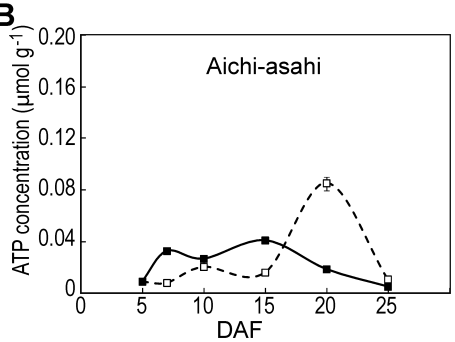

E

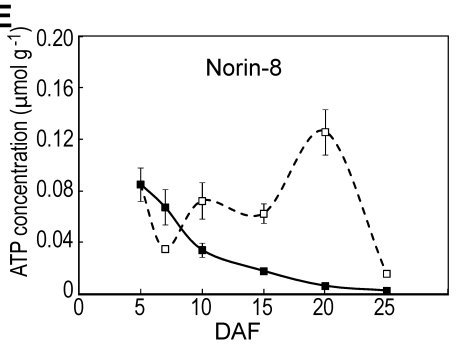

H

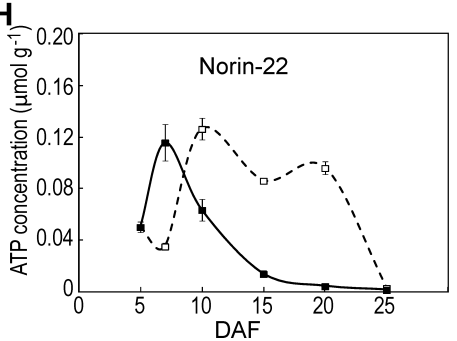

c

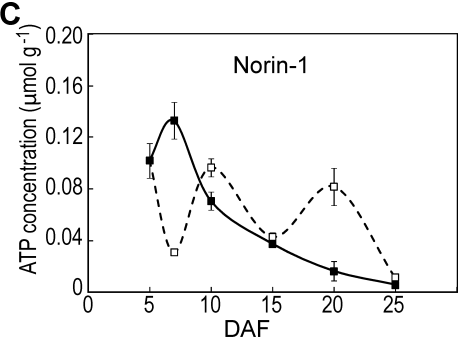

$F_{-}$

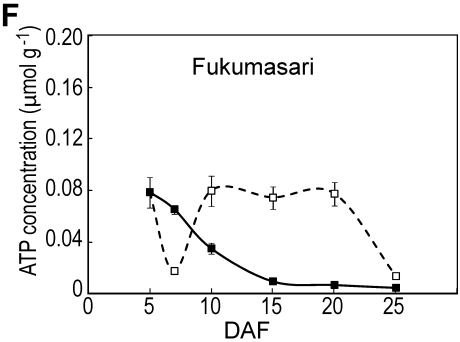

- - $\square$ - Normal Temperature

$\rightarrow-$ High Temperature

Figure 2. Time course of ATP content fluctuations during seed development in normal and high temperature conditions. The ATP content was measured in six immature seeds from Shinriki (A), Aichi-asahi (B), Norin-1 (C), Nikomaru (D), Norin-8 (E), Fukumasari (F), Ginbouzu (G), and Norin-22 $(\mathrm{H})$ at 5, 7, 10, 15, 20, and 25 DAF. The open and filled circles represent seeds grown in normal and high temperature conditions, respectively. The error bars represent the standard errors.

main cultivars and many cultivars are the progeny of Norin-22 (Figure 3). We also found that Nipponbare, which is also derived from Norin-22, was very sensitive to high temperature stress (She et al. 2010a). Our observations suggest that the poor high temperature stress tolerance of Koshihikari and Nipponbare were attributable to their origins in Norin-22.

Shinriki produced a large proportion of normalshaped grains even when they were grown in high temperature conditions, although the yield was slightly lower because of reduced grain size (Tables 1 and 2). These results suggest that Shinriki has good high temperature tolerance.

The seed development process in Taichung-65 was found to be inhibited by high temperature stress, which resulted in a significant reduction in the grain yield. However, the proportion of grains with defective features was not increased greatly by high temperature stress (She et al. 2010a). We found that Kimmaze was tolerant of the high temperature stress. The pedigree chart indicates that both Taichung-65 and Kimmaze are derived from Shinriki (Figure 3). These results suggest that Shinriki has an important trait that prevents grains from producing defective characteristics in high temperature stress conditions.
Previously, it was suggested that an inadequate ATP content caused the appearance of defective characteristics in Nipponbare grains (She et al. 2010a). In the current study, we found that the grains of all cultivars had a significantly lower ATP content in high temperature stress conditions (Figure 2). The ATP content of Norin-22 seeds was clearly decreased by high temperature stress conditions. By contrast, Shinriki maintained a relatively high level ATP content throughout the seed development stages (Figure 2). ATP is required for multiple cell functions including plant growth and storage substance accumulation. The presence of phenotypic changes such as the appearance of small grains and white core endosperm was strongly linked to the reduced ATP content of the developing seeds. We suggest that inadequate supply of ATP led to inefficient grain filling, which resulted in a reduced accumulation of storage substances.

The seed development process is promoted in the high temperature conditions (Yamakawa et al. 2010). The ATP content had two peaks at $10 \mathrm{DAF}$ and 20 DAF in the normal condition. The second peaks were shifted to the $15 \mathrm{DAF}$ in the high-temperature conditions, and overall these peak heights were smaller than those in the normal conditions (Figure 2). We detected the correlation 


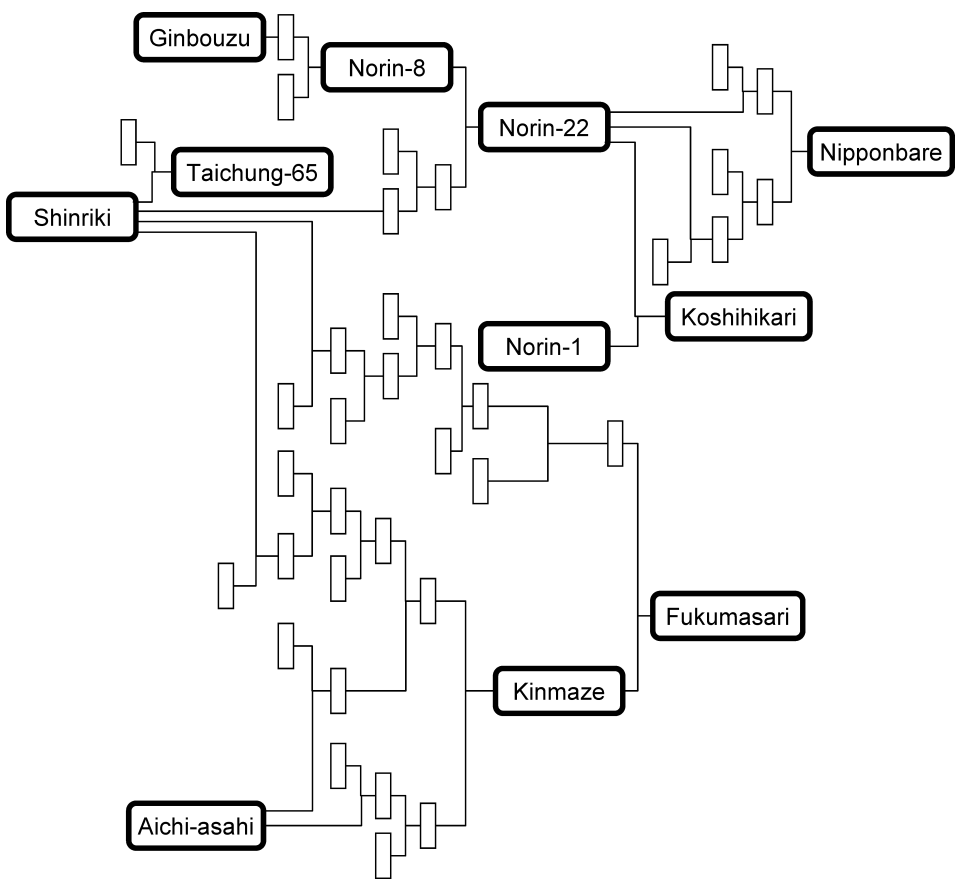

Figure 3. Simplified pedigree chart of the representative rice cultivars. The open boxes represent the corresponding cultivars while the lines indicate direct inheritance and relationships with progeny. Data on the breeding processes were derived from the database established by the National Institute of Crop Science (http://ineweb.narcc.affrc.go.jp).

between the proportion of the perfect grain and the ratio of the level of the ATP contents at the second peaks in the high temperature conditions to those in the normal conditions (Figure 4). This suggests that reduction of ATP content corresponding to the second peak causes insufficient grain filling in the high temperature conditions.

Nikomaru is reported to be a high temperaturetolerant cultivar in the actual field (Shirato et al. 2007). In our experiment, we found that Nikomaru seeds contained higher levels of ATP throughout the seed development stages in normal temperature conditions (Figure 2). This suggests that Nikomaru has an unknown trait, which enables to provide a high ATP supply in developing seeds. However, the ATP levels of the grains declined significantly when Nikomaru seeds were grown in high temperature stress conditions (Figure 2). This suggests that Nikomaru is also sensitive to high temperature stress, although our experimental conditions were intense for any rice cultivars to grow compared with actual field conditions. It has been shown that Nikomaru accumulates a large amount of non-structural carbohydrates, which is important for expression of the high temperature tolerance (Morita et al. 2008). We suggest that Nikomaru was tolerant of high temperature stress in actual field conditions because the effect of high temperature stress appeared to be more moderate in this cultivar.

In conclusion, we developed a model to account for the appearance of defective phenotypes with high

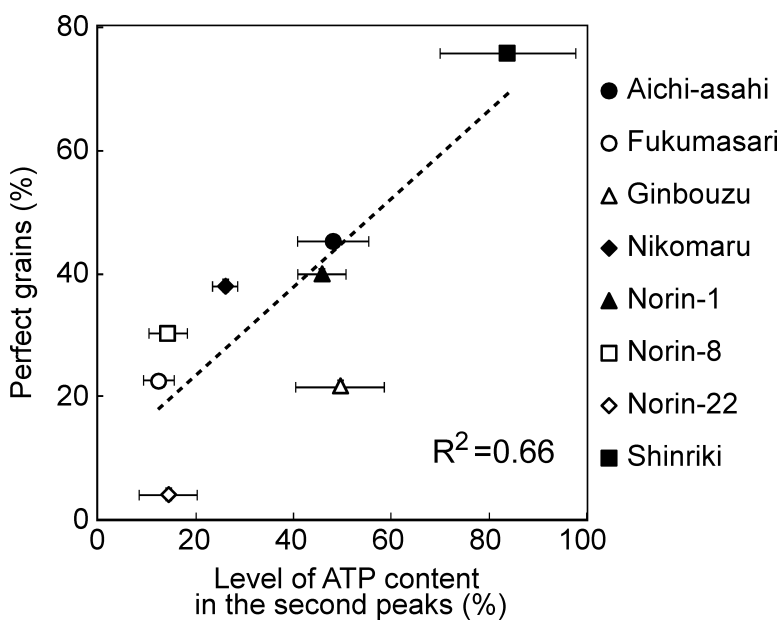

Figure 4. Correlation between the proportion of perfect grains and the level of the ATP contents at the second peak in the high temperature condition compared to those in the normal temperature. Horizontal axis shows the ratio of ATP content at 15 DAF in the high temperature conditions to those in the normal conditions (20 DAF). Ratio of perfect grain developed under high temperature conditions indicates as vertical axis.

temperature stress. As suggested previously, an adequate supply of ATP is very important for grain productivity. The ATP supply is inhibited significantly by high temperature stress throughout the seed development stages, which results in the formation of grains with defective characteristics. Shinriki exhibited fewer defective characteristics without a major reduction in the ATP content of the developing seeds, which suggests 
there is a relationship between low ATP content and the appearance of defective endosperm characteristics. Therefore, it is suggested that a rice cultivar with an adequate ATP supply during seed development will be very useful for breeding a high temperature-stress tolerant cultivar.

\section{Acknowledgements}

This work was support by a Grant from the Ministry of Agriculture, Forestry and Fisheries of Japan (Genome for Agricultural Innovation, IPG-0022).

\section{References}

Kimmich GA, Randles J, Brand JS (1975) Assay of picomole amounts of ATP, ADP, and AMP using the luciferase enzyme system. Anal Biochem 69: 187-206

Morita S, Tamura K, Nakano H, Kitagawa H, Sakai M, Takahashi M (2008) Contribution of higher amounts of non-structural carbohydrates (NSC) in stems at full heading to better ripening of heat-tolerant rice cultivar nikomaru. Jpn J Crop Sci 77(suppl. 2): 198-199, in Japanese

Peng S, Huang J, Sheehy JE, Laza RC, Visperas RM, Zhong X, Centeno GS, Khush GS, Cassman KG (2004) Rice yields decline with higher night temperature from global warming. Proc Natl Acad Sci USA 101: 9971-9975

She KC, Kusano H, Yaeshima M, Sasaki T, Satoh H, Shimada H (2010a) Reduced rice grain production under high-temperature stress closely correlates with ATP shortage during seed development. Plant Biotechnol 27: 67-73
She KC, Kusano H, Koizumi K, Yamakawa H, Hakata M, Imamura T, Fukuda M, Naito N, Tsurumaki Y, Yaeshima $M$, Tsuge T, Matsumoto K, Kudoh M, Itoh E, Kikuchi S, Kishimoto N, Yazaki J, Ando T, Yano M, Aoyama T, Sasaki T, Satoh H, Shimada H (2010b) A novel factor FLOURY ENDOSPERM2 is involved in regulation of rice grain size and starch quality. Plant Cell 22: 3280-3294

Shirato Y, Fukushima A, Ogata N, Ohara T, Kawashima K, Harada H, Yamada T, Furuita H, Toshio O (2007) Impact of global warming on agriculture, forestry and fisheries and possible countermeasures in Japan. in: Research and Development in Agriculture, Forestry and Fisheries No. 23, ed. Agriculture, Forestry and Fisheries Research Council, MAFF Japan. Ministry of Agriculture, Forestry and Fisheries (MAFF), Japan, pp. 3-8

Tashiro T, Wardlaw IF (1991) The effect of high temperature on kernel dimensions and the type and occurrence of kernel damage in rice. Aust J Agric Res 42: 485-496

Terashima K, Saito Y, Sakai N, Watanabe T, Ogata T, Akita S (2001) Effects of high air temperature in summer of 1999 on ripening and grain quality of rice. Jpn J Crop Sci 70: 449-458

Yamakawa H, Hirose T, Kuroda M, Yamaguchi T (2007) Comprehensive expression profiling of rice grain filling-related genes under high temperature using DNA microarray. Plant Physiol 144: 258-277

Yamakawa H, Hakata M (2010) Atlas of rice grain fillingrelated metabolism under high temperature: joint analysis of metabolome and transcriptome demonstrated inhibition of starch accumulation and induction of amino acid accumulation. Plant Cell Physiol 51: 795-809

Zakaria S, Matsuda T, Tajima S, Nitta Y (2002) Effect of high temperature at ripening stage on the reserve accumulation in seed in some rice cultivars. Plant Prod Sci 5: 160-168 